\title{
Sul comportamento di alcuni innesti di occhi nelle larve di Discoglossus pictus.
}

\author{
Per il
}

Dott. 0. Anastasi.

(Istituto di anatomia comparata di Palermo, diretto dal Prof. Raffaele.)

Con tavole IX-XI.

Eingegangen am 16. April 1913.

In questo ultimo decennio, si sono ottenuti interessanti risultati col metodo degli innesti fra girini di rane.

Il BorN nel suo lavoro: Über Verwachsungsversuche mit Amphibienlarven ha trattato e svolto esaurientemente questo argomento, giovandosi di innesti fatti in tutte le maniere possibili, per avere sott'occhio le modificazioni che subivano $i$ varii organi ed $i$ varii tessuti cosi riuniti.

Ho cercato applicare questo metodo alla soluzione del quesito seguente:

Dato che la vescicola ottica e la porzione di ectoderma a lei prossima contengono in potenza le varie parti che dovranno costituire l'occhio, vedere, messi con artificii di tecnica parti di due vescicole ottiche a contatto, quale potere regola lo sviluppo dei varii tessuti che danno luogo all'organo della visione.

Per la soluzione del quesito propostomi, cercai di innestare porzione del capo di un girino sul capo di un altro girino: non mi fu dato però ottenere l'attecchimento dell' innesto, per la rapidità con la quale questo cade in necrosi subito dopo la separazione dall' orgaganismo intero.

Allora ricorsi ad un espediente: asportavo dal capo di una larva una calotta, comprendente una parte della v. o.; lo stesso facevo su di un'altra e facevo combaciare le due superficie scoperte. 
Parlerò appresso della tecnica da me seguita: faccio solo qui notare che le mie esperienze ebbero pieno successo per la maniera facilissima con cui questi innesti attechivano.

Purtroppo, malgrado questa facile adesione degli innesti, due grandissime difficoltà e'era da superare.

1. Non ostante mi servissi prima della lente binoculare per cercare il posto dove il coltello doveva cadere, per dividere la vescicola ottica, in molti casi succedeva che il taglio cadeva in maniera da portar via col pezzo asportato tutta la vescicola ottica, o cadeva molto lontano in maniera che gli innesti venivano senza occhi nel primo caso o con $\dot{i}$ due occhi separati da un buon tratto di tessuto nel secondo caso. Di questi innesti non potevo servirmi per il mio studio.

Ho sormontato questa difficoltà facendo di questi innesti a migliaia e scegliendo quelli che servivano al mio scopo.

$2^{\circ}$. L'altra non meno grande difficoltà m'era data dal fatto che gli innesti avevano poca vitalità.

Il loro potere di resisteuza diminuiva man mano che venivano sviluppandosi ed avevo delle vere ecatombi di innesti, tanto che, per avere pochi stadii perfetti di avanzato sviluppo, dovetti sacrificare innumerevoli larve.

\section{Materiale di esperienze e tecnica.}

Mi son servito di larve di Discoglossus pictus sulla gran quantità delle quali io potevo contare per il mio lavoro.

Strumenti: un coltellino di Graffe affilatissimo, una forbice a punte acutissime e taglienti, una pinzettina, un pennello di vaio.

Una lastra di vetro coperta con carta bibula faceva da tavolo operatorio. Su questo io mettevo un quadratino di carta bibula e su di esso facevo cadere con una pipetta le due larve da operare.

Mi son servito sempre di larve appena venute fuori dall' uovo perciò al primissimo stadio di sviluppo; ho quindi operato su vescicole ottiche primitive.

Partendo poi dal concetto, di volere cogliere la vescicola ottica in tutti i suoi punti ed in tatte le sue direzioni, ho diviso la testa del girino in un quadrante di cui faceva centro la fossetta adesiva e facevo cadere $i$ tagli lango le direzioni dei suoi raggi; in tal maniera, ebbi innesti che si riunivano per la testa dalla porzione anteriore, dalla porzione posteriore e dalla porzione centrale: sempre i tagli però passavano per la vescicola ottica. 
Occorreva pertanto nei maneggi delicati di non ledere il girino in nessuna parte del corpo: nel caso in cui ciò avreniva, bisognava buttare l'innesto.

Fatte le incisioni, col pennellino imberuto di acqua, portavo via i pezzettini della parte tagliata, e quindi, aiutato da una debole corrente di acqua, che facevo gocciolare col pennello, mettevo i due girini a contatto per la superficie del taglio. Arvenuta la adesione, pigliavo il pezzetto di carta bibula che li eonteneva e lo posavo in una camera umida. $\dot{E}$ interessante la disposizione di questa camera umida, perchè i girini morirebbero se non fossero immersi in acqua.

Su un piatto pieno di acqua, è posta una vaschetta di cristallo capovolta coperta di carta bibula che pesca nell'acqua del piatto.

E su questa carta bibula che io posavo i miei innesti. Il tutto ricoprivo con un'altra vasca più grande capovolta.

Dopo due giorni rimovevo questi innesti e li mettevo senża scuoterli in grandi vasche piene di acqua di fonte; ivi si distaccavano dalla carta e cominciavano a nuotare per la vaschetta (v. Fig. 1 e 2). Fatta la pulizia delle sostanze estranee, avevo cura tre, quattro volte al giorno di rimuovere l'acqua, togliere gli innesti morti e quelli la cui vitalità mi sembrava compromessa.

Un giorno dopo dall' innesto attechito, dopo tre quindi dall' operazione, cominciai a fissarli giorno per giorno in sublimato e servendomi della lente binoculare selezionavo quelli inservibili ed avero cura di quelli che mostravano un solo occhio nel punto medio dell'innesto. Tutti venivano poi inclusi in paraffina, tagliati al microtomo in serie e colorati con i varii colori.

Come confronto per lo sviluppo dell' occhio, ho fatto una serie di preparati di larve normali dal $1^{\circ}$ al $15^{\circ}$ giorno di sviluppo.

Nella esposizione dei reperti anatomici riunirò in unica descrizione tutte le particolarità degne di nota che io ho trovato in innesti dello stesso stadio di sviluppo.

Esaminando la serie dei preparati a cominciare dagli stadii primitivi ho rilevato le seguenti interessanti particolarità:

Innesto A (v. Fig. 3):

(Corrisponde al $3^{\circ}$ giorno dopo l'innesto: venne fissato in sublimato acetico e colorato in serie con ematossilina di Bizzozzero.)

Le vescicole ottiche, comprese in una stessa cavità sono anteriormente, in vicinanza all' epitelio ectodermico, unite da un ponte di 
cellule, la cui struttura è uguale a quella delle cellule delle vescicole ottiche. Queste sono separate da un solco mediano che va fino al ponte sopra cennato.

L'epidermide, formata da due strati di cellule si continua lungo il punto di adesione dei due innesti, senza nessuna alterazione nella disposizione e nella forma dei suoi elementi.

In prossimità di una delle due vescicole ottiche l'ectodermo in proliferazione accenna alla formazione della vescicola cristallinica.

Corrispondentemente all' altra vescicola ottica non esiste nel tratto di epidermide a lei prossimo questa differenziazione in un abbozzo cristallinico.

Le rescicole ottiche sono formate da tre a quattro strati di cellule omogenee fusiformi: lo strato pit̀ prossimale assume una forma più fusata.

Le due vescicole ottiche sono contenute in una cavità piriforme i cui bordi sono fatti a spese della sostanza nervosa e la cui parte più ristretta, il futuro peduncolo ottico, è costituito per metà da sostanza appartenente ai due diversi girini messi a contatto.

Da questa cavità, per un canale, si passa alla cavità cerebrale la quale, essendo formata dall' unione delle due cavità appartenenti ai due innesti, piglia una caratteristica forma di cifra 8 .

Innesto B (v. Fig. 4).

E uno stadio più avanzato del precedente. La cavità che accoglie le due rescicole è chiusa, gli elementi che costituiscono le rescicole ottiche più sviluppati e cresciuti in numero. Anche la vescicola cristallinica ha seguito il suo accrescimento.

$\dot{\mathrm{E}}$ degno di nota in questo innesto, un fatto importantissimo.

Corrispondentemente alla v. o. di sinistra un po' inclinata verso quella di destra, non esiste nell' ectoderma la modificazione che subiscono le sue cellule per la formazione del cristallino, invece dal margine sinistro dello abbozzo cristallinico della vescicola ottica di destra, si parte uno zaffo di cellule simili a quelle del cristallino e vanno alla cavità della v. o. di sinistra. É probabile che esse, daranno appresso origine alla lente di questa parte della v. 0.

\section{Innesto $\mathrm{C}$ (v. Fig. 5).}

Qui notiamo la disposizione identica alla precedente, delle vescicole ottiche e degli elementi che le costituiscono. La rescicola cristallinica del lato sinistro è completa e, come nell'innesto prece- 
dente, dalla porzione corrispondente alla v. o. di destra, si continua une serie di cellule che va a finire nella cavità di questa v. 0.

\section{Innesto D (v. Fig. 6).}

In questo innesto troviamo un occhio provvisto di tutte le sue parti in istato di avanzato sviluppo, in una insenatura del quale pochi elementi appartenenti all'occhio dell' embrione adiacente si sono sviluppati prendendo la forma di un occhio piccolo ed incompleto.

Qui i due occhi non si sono trovati vicini che per le parti opposte e i cristallini si sono sviluppati da parti opposte l'una all'altra. Anzi è degno di nota il fatto che, mentre nella vescicola ottica completa il cristallino si è sviluppato dalla porzione vicina di epidermide, in quello atrofico i cui elementi si trovarano immersi nel tessuto connettivo, la formazione del cristallino dovette avvenire a spese di uno zaffo epiteliale che dal punto di combaciamento dei due innesti, arriva fin quasi in prossimità di questo secondo occhio.

\section{Innesto F (v. Fig. 7).}

Le due rescicole ottiche simmetricamente disposte, danno l'idea di un grande occhio fatto per le due metà destra e sinistra a spese delle due vescicole appartenenti alle due larve.

Gli elementi grandi e stratificati appartenenti alle retine dei due occhi, sono divise da un setto di pigmento che arriva fino alla parte inferiore del calice ottico: anteriormente a questo punto avviene la formazione di un ponte di cellule retiniche, appartenenti alle vescicole ottiche, ehe senza alcun limite di passaggio, si confondono fra di loro. Dalla parte posteriore, questo sepimento pigmentale cireonda le due mezze rescicole ottiche ed anteriormento circonda le due porzioni delle vescicole ove comincia a formarsi la parte ciliare della retina.

Tra queste due porzioni simmetriche, i due cristallini appartenenti ai due occhi si sono fusi, formando una rescicola cava separata da un tramezzo epiteliale molto ben manifesto. Anteriormente, tra l'epidermide e l'occhio un ammasso di cellule connettivali si stende dall'una all'altra parte di questa doppia vescicola. $\dot{\mathrm{E}}$ già cominciata la formazione della cornea.

Innesto Gr (v. Fig. 8):

É uno stadio ancor più avanzato del precedente che lo copia fedelmente e mette meglio in mostra il modo di comportarsi delle due vescicole ottiche. 
Il sepimento coroideale che divideva nel precedente le cellule appartenenti all' una e all'altra retina, non esiste e la coroide unita posteriormente in maniera da non fare osservare nessuna linea discontinua, racchiude gli elementi delle due $\nabla .0$.

La vescicola cristallinica unica, ma col setto epiteliale che la divide in due logge, è formata da cellule cubiche anteriormente: posteriormente le cellule vanno assumendo la forma fusata propria delle cellule che daranno origine alle fibre del cristallino.

\section{Innesto $H$ (r. Fig. 9).}

La stessa posizione delle v. o. - Il cristallino è sviluppato di più del precedente e vi si notano due nuclei separati di fibre appartenenti alle due vescicole cristalliniche unite e formatesi quindi separatamente a spese delle cellule proprie. Posteriormente la coroide segue le insenature delle due vescicole, però mentre la chiusura è avrenuta in quella di destra, in quella di sinistra, trovasi ancora ampio il canale ottico.

Innesto $I$ (v. Fig. 10).

Questo innesto è di una chiarezza degna di nota. Le due retine ormai sviluppate sono circondate dalla coroide che posteriormente si fa unica per entrare nella insenatura formata dai due calici ottici fino a toccare la faccia posteriore del cristallino.

Questo è unico formato a cifra 8 per l'unione dei due cristallini che sono fra loro saldati per un ponte di cellule che passa dall'una all' altra vescicola.

A destra comincia a notarsi il disgregamento delle cellule che formano la parete sinistra della cristalloide. $\dot{E}$ evidentissima qui la tendenza di due setti intermedii a scomparire per dar luogo alla formazione di un'unica capsula perilenticulare e quindi ad un unico cristallino per il fondersi delle masse.

Innesto L (v. Fig. 11).

La fusione delle masse cristalliniche notata già come incipiente nell'innesto precedente; è qui molto inoltrata.

I setti intermedii sono scomparsi e la capsula, unica e senza alcun segno di passaggio dagli elementi dell' una a quelli dell'altra vescicola cristallinica, contiene i due cristallini che ancora sono ben divisi e riconoscibili. Niente évvi di notevole nella costituzione dei calici ottici più del precedente, ove si noti una particolarità nello sviluppo completo della retina e nella struttura della cornea, la quale 
partendo dall'una e dall' altra metà delle vescicole ottiche, arrivata a metà si mette a contatto con la superficie anteriore del cristallino.

Innesto M (v. Fig. 12).

$\dot{E}$ una sezione tagliata di fronte dello stesso stadio del precedente.

Innesto $\mathrm{N}$ (v. Fig. 13).

Uno stadio ancora più avanzato del precedente. Appare chiara la fusione delle due masse cristalliniche circondate da unica capsula di elementi uguali e continui. Gli elementi retinici fusi nel loro punto di contiguità lasciano ancora trasparire la diversa origine da due rescicole adiacenti. La cornea si profila diritta come un ponte di cellule che va dall' una all'altra parte senza nessun punto di contatto colla porzione anteriore della capsula cristallinica. Avanzati di sviluppo sono le formazioni dell'iride.

\section{Innesto $O$ (v. Fig. 14).}

$\grave{\mathrm{E}}$ un innesto in cui $\mathrm{i}$ due occhi non sono capitati proprio giustapposti ma spostati un poco in maniera che mentre dell' uno si nota una sezione nel punto centrale, dell'altra appare una piccola porzione. Pure gli stessi fatti dell'innesto precedente si mostrano evidentissimi e si notano le due porzioni di nucleo cristallinico e le due porzioni di retine che messe a contatto fondono i propri elementi e danno l'illusione che si tratti di un organo unico.

\section{Innesto $\mathrm{P}$ (v. Fig. 15).}

Se un piccolo solco non indicasse la divisione delle due retine ed un sottile cumulo di pigmento non rivelasse lo assorbimento della. coroide insinuata fra gli elementi appartenenti all'una e all'altra retina, si direbbe che questo sia un occhio unico di un unico individuo, tanto gli elementi retinici dell' una vescicola ottica si continuano strato a strato senza alcun limite di demarcazione con quelli dell'altro

Ed a mantenerci nella stessa illusione sta il fatto che i due cristallini, fusi insieme, ne formeranno un solo quasi che le fibre cristalliniche siano il prodotto della proliferazione degli elementi cristallinici di una sola vescicola.

Sono molto sviluppati gli elementi che costituiscono l'iride; uno strato di cellule situate a ponte fra l'uno e l'altro processo irideo ci mostra la formazione della cornea.

\section{Innesto $Q$ (v. Fig. 16).}

Che siano due gli occhi rappresentati da questa sezione ce lo dicono le sezioni che la precedono e quelle che la seguono: a prima 
vista è facile confonderla con quella in un solo occhio tanto le due parti si sono fuse insieme a formarne uno.

Gli elementi della retina, stratificati come quelli di un occhio adulto, non presentano differenza alcuna nelle due parti venute a contatto; cosi il cristallino (fusione dei due cristallini), sembra essere il prodotto di una sola vescicola cristallinica.

In corrispondenza alla porzione sinistra dell'occhio si nota la presenza di un vaso che, attraversata la coroide e gli elementi retinici, va fino alla porzione posteriore del cristallino.

Gli elementi che costituiscono la cornea passano dall'uno all' altro occhio sopra le corrispondenti parti che costituiseono la retina, direttamente senza alcun punto di contatto con la porzione anteriore delle capsule della lente. Uno strato di cellule bene appariscenti, unite in linea copre la coroide: sono le cellule della sclera: esse continuano quelle della cornea e circondano tutto l'occhio.

Innesto $R$ (v. Fig. 17).

Ho voluto mettere qui in evidenza il fatto importantissimo di due occhi uniti fra di loro, i quali, malgrado siano separati dalle membrane della coroide, pure presentano una particolarità degnissima di nota.

Le cellule che formeranno la cornea passano dall' uno all' altro occhio formando una sola curva che non presenta nessun punto di contatto con le due v. o.

Innesto $\mathrm{S}$ (v. Fig. 18).

Rappresenta questa microfotografia (Fig. 18) la porzione terminale di un innesto là dove $i$ due ochi sono fusi in uno e dalla porzione posteriore di una vescicola ottica si parte il nervo ottico che mette in unione le due vescicole con la porzione adiacente della vescicola cerebrale.

Cominciano a formarsi qui le porzioni di tessuto cartilagineo ehe darà poi luogo alla formazione dello scheletro dell'orbita.

Disgraziatamente oltre questo stadio di sviluppo non mi è stato possibile tenere ancora in vita gli innesti e quindi di seguirne lo sviluppo ulteriore.

Ma a me pare che da quello che emerge dalle osservazioni sopra descritte si possa ben venire a delle considerazioni che rispondano alla domanda che mi son fatta fin dall'inizio di questo mio lavoro. 


\section{Conclusioni.}

Abbiamo visto come le due vescicole primitive, messe a contatto per parti cruente di esse, vengono a disporsi in maniera che gli elementi di una retina si uniscano a quelle dell'altra in un modo cosi uguale da non notarsi alcuna discontinuità fra le due porzioni di retine a contatto, nè alterazione alcuna per il numero, nè per la natura degli elementi stessi. Abbiamo notato che, quando le due retine erano divise dallo strato di pigmento, un ponte di cellule retiniche andava dall'una all'altra v. o. unendo i vari strati delle cellule dell' una retina e quelle dell' altra.

Ambedue le vescicole ottiche giacciono nella stessa cavità che è fatta a spese di due mezze cavità simmetriche appartenenti all uno ed all' altro occhio.

Sembra che quando le due vescicole cristalliniche vengono a contatto, allora le due porzioni per cui avviene l'addossamento, si fondono, formando un tramezzo. Questo tramezzo a poco a poco pare che vada disfacendosi, poichè non lo ritroviamo in altri stadii più avanzati, quelli cioè in cui le masse cristalliniche, più sviluppate, si addossano l'una all'altra. Dico pare, perchè nonostante la cosa sembri evidente, pure non possiamo con certezza asserirlo, dato che non è possibile avere di ano stesso innesto, i diversi stadii che ci potessero dare le diverse fasi evolutive dell'unione dei due cristallini.

Anche la distinzione in due nuclei separati scompare col progredire dello sviluppo, poichè le fibre dell' uno e dell'altro cristallino si uniscono infine a formarne un solo ammasso come se si trattasse di unica formazione; epperò sinó ad un certo svilluppo si notano i due nuclei ciascuno involto dal proprio strato di fibre, poi questa divisione sparisce.

Gli elementi che costituiscono le cornee dei due occhi, convergono dapprima verso un punto della capsula anteriore del cristallino, forse in corrispondenza del punto dove avviene la saldatura delle due capsule cristalliniche; ma poi si stacca questa aderenza e le cellule si distendono in unica linea a formare un ponte che va dall'involucro sclérale dell'una metà a quello dell'altra metà.

Cosi le formazioni ciliari e iridee sono fatte a spese dei due mezzi occhi che si vengono a trovare a contatto e si sviluppano insieme a formare un tutto unico armonico come se si trattasse delle due porzioni simmetriche di una stessa vescicola ottica. 
Il nervo ottico viene pure ad essere formato da porzioni di tessati appartenenti a sostanza delle due vescicole cerebrali, che anch' esse si saldano in maniera da circondare una cavità che è il prodotto dell'unione delle due cavità appartenenti alle due vescicole venute in contatto. E questo si nota maggiormente nel periodo più aranzato di sviluppo in cui a due vescicole ottiche adulte, fa capo un solo nervo ottico che va a finire nel punto di combaciamento delle due porzioni adulte di cervello.

E la sclera? E la coroide?

Anche esse pare seguono la legge generale di sviluppo pocanzi accennata. Ed è fuor di dubbio che si può in questo caso rispondere al quesito che mi sono proposto nell 'inizio di questo mio lavoro dicendo che: le, due vescicole ottiche venute a contatto per parti cruente di esse tendono a regolarsi in modo da formare un unico occhio comprendente le porzioni sia dell' una che dell'altra vescicola ottica e che questi tessuti si sviluppano in guisa da formare un unico organo che si sviluppa nella stessa maniera come se gli elementi appartenessero non a due individui differenti ma ad un solo individno.

\section{Zusammenfassung,}

Werden zwei primäre Augenblasen mit angefrischten Teilen derselben in Kontakt gebracht, so ordnen sie sich derartig an, daß die Elemente der einen Netzhaut sich.mit denen der andern so gleichförmig vereinigen, daß keinerlei Diskontinuität zwischen den zwei in Kontakt befindlichen Netzhautportionen noch irgendwelche Veränderung nach Zahl und Beschaffenheit der Elemente selbst wahrzunehmen ist.

Wenn die beiden Linsenblasen in Kontakt kommen, dann verschmelzen die beiden Portionen, durch die die Anlagerung erfolgt, miteinander und bilden eine Scheidewand, die sich nach und nach aufzulösen scheint. Die Fasern der einen und der andern Linse vereinigen sich schließlich zu einem Ganzen, wie wenn es sich um ein einziges Gebilde handelte.

Die die Hornhäute der beiden Augen bildenden Elemente konvergieren zuerst gegen einen Punkt der vorderen Linsenkapsel, wohl entsprechend der Stelle, wo die Verlötung der beiden Linsenkapseln erfolgt; dann aber löst sich diese Verwachsung und die Zellen dehnen sich in einer einzigen Linie zur Bildung einer Bricke, welche von der skleralen Hïlle der einen Hälfte zu der der andern Hälfte zieht.

Die Ciliarkörper- und Irisbildung erfolgt auf Kosten der zwei halben Augen, die in Kontakt kommen und sich zusammen zu einem einzigen harmonischen Ganzen entwickeln, als ob es sich um die beiden symmetrischen $\mathrm{Ab}$ schnitte einer und derselben Augenblase handelte.

Der Sehnerv wird ebenfalls durch Gewebsportionen gebildet, die zur Substanz der beiden Hirnblasen gehören, welche gleichfalls in der Weise verwach- 
sen, daß sie eine Höhle umgeben, die das Produkt der Vereinigung der zwei Höhlen der beiden in Kontakt gekommenen Blasen ist.

Und die Sklera? Und die Chorioidea?

Auch sie scheinen dem allgemeinen, soeben angedeuteten Entwicklungsgesetz zn folgen. Es steht außer Zweifel, daß sich in diesem Fall sagen läßt, daß die mit angefrischten Partien in Kontakt gekommenen Augenblasen sich derart zu verhalten streben, daß sie ein einziges, die Portionen sowohl der einen wie der andern Augenblase umfassendes Auge bilden und da $B$ diese Gewebe sich zu einem einzigen Organ ausbilden, das sich in der gleichen Weise entwickelt, wie wenn die Elemente nicht zwei verschiedenen Individuen, sondern nur einem Individuum angehörten. 


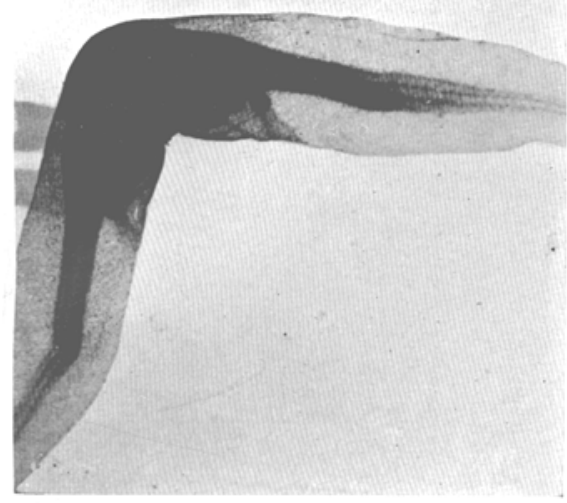

Fig. 1

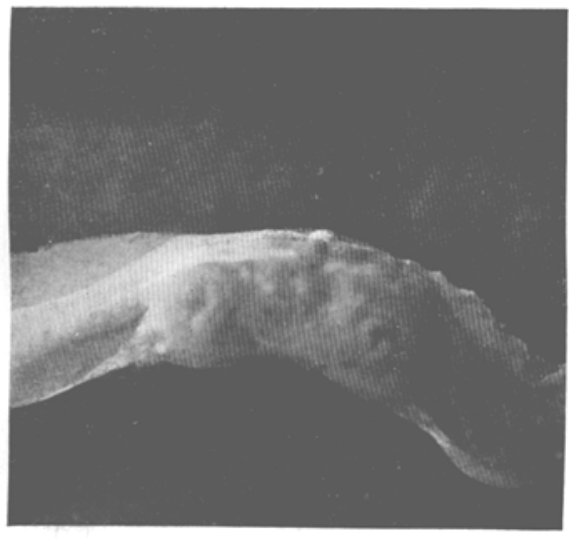

Fig. 2

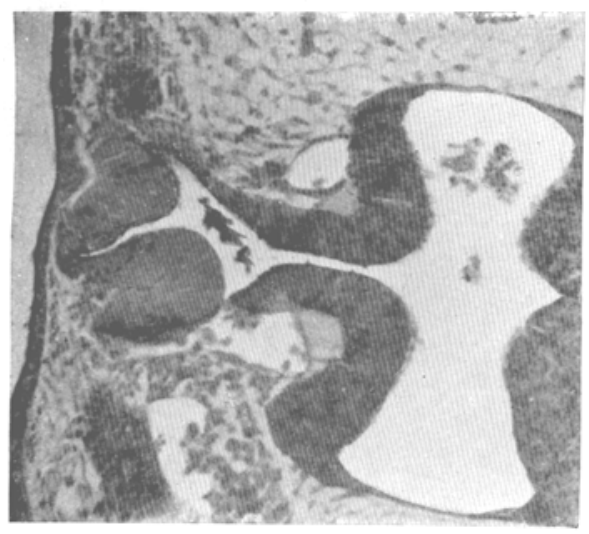

Fig. 3

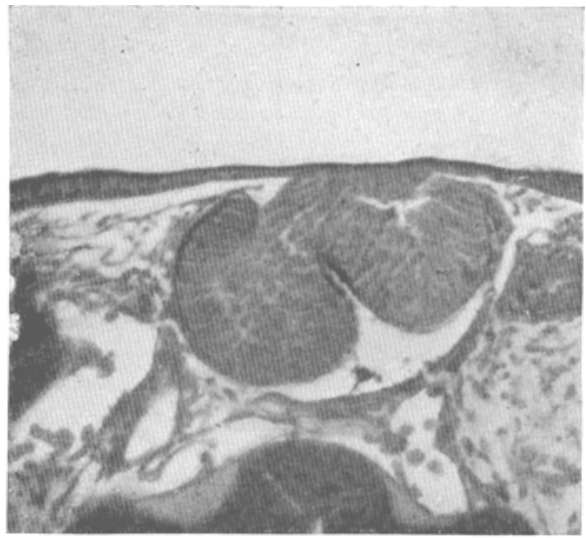

Fig. 4

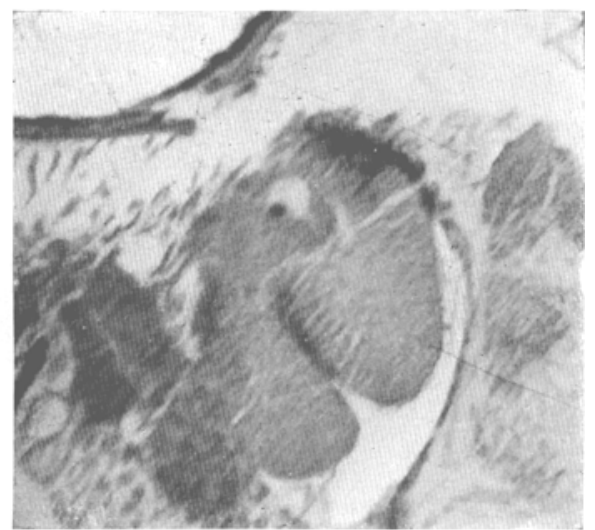

Fig. 5

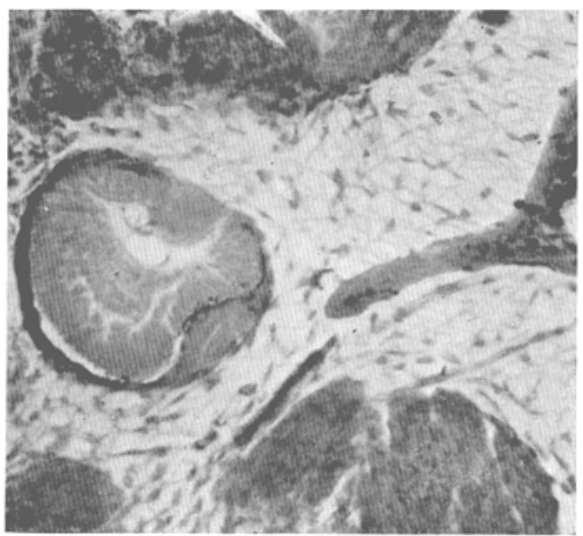

Fig. 6

Anastasi.

Verlag von Wilhelm Engelmann in Leipzig und Berlin. 


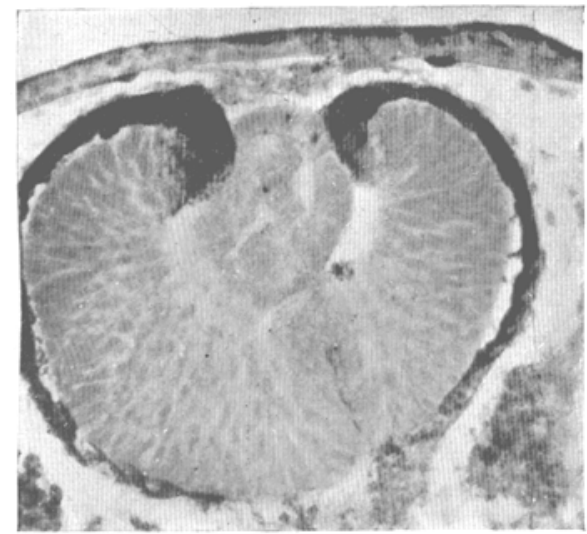

Fig. 7

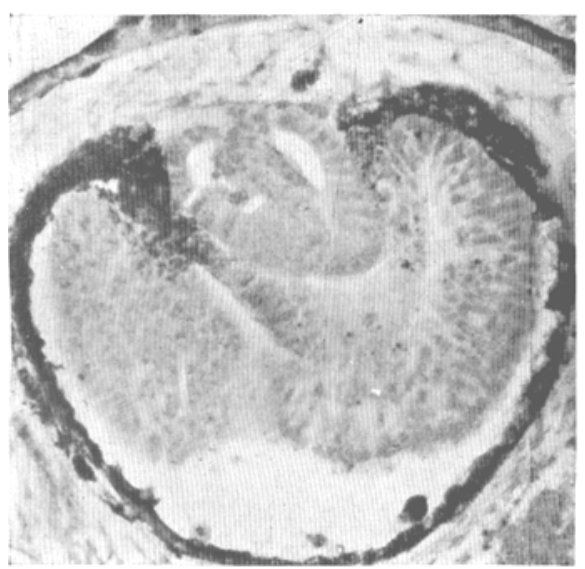

Fig. 8

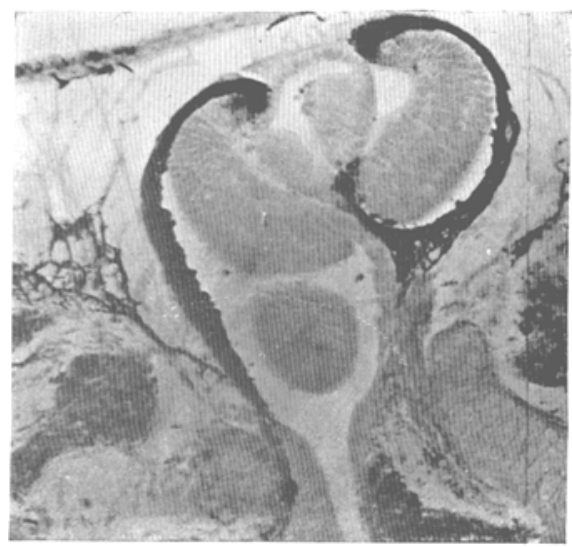

Fig. 9

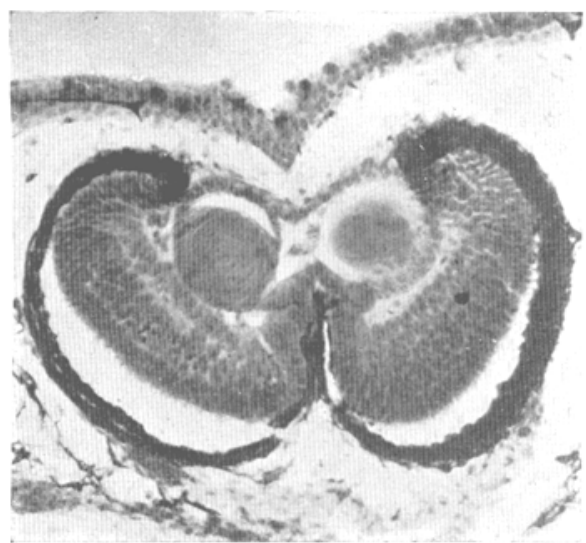

Fig. 10

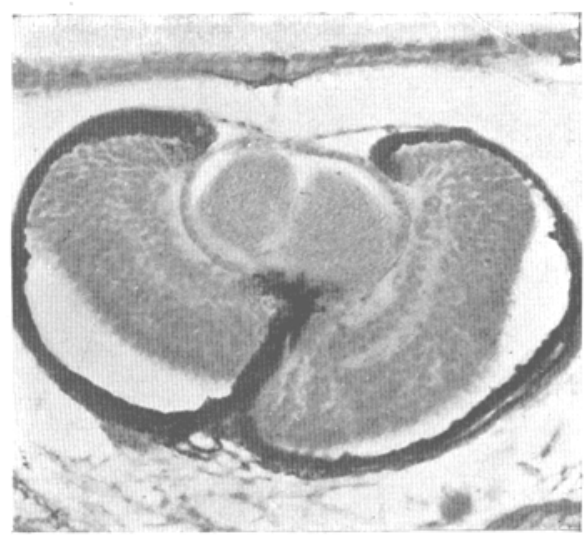

Fig. 11

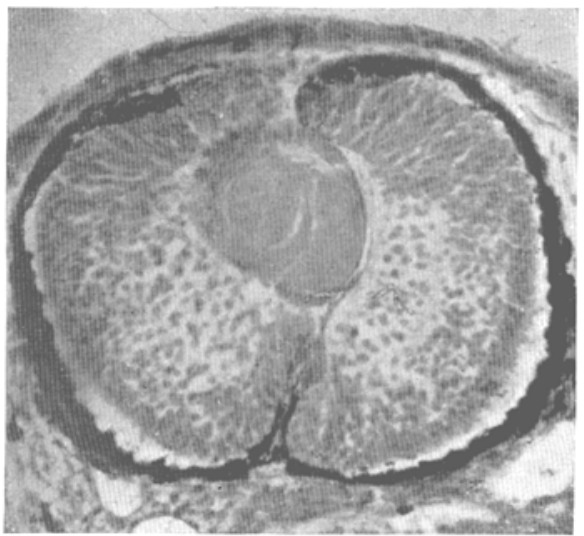

Fig. 12 


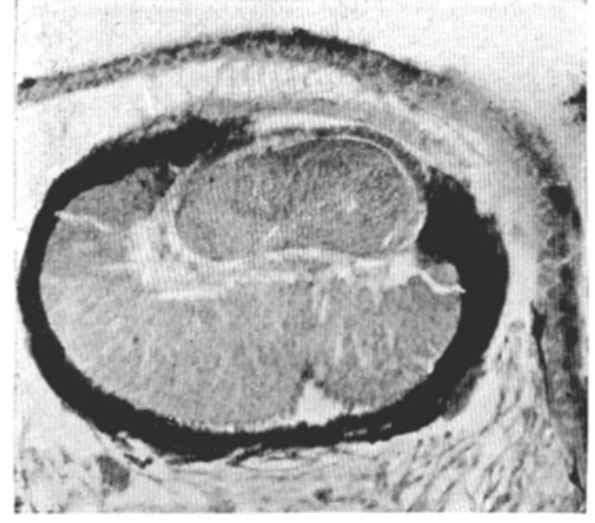

Fig. 13

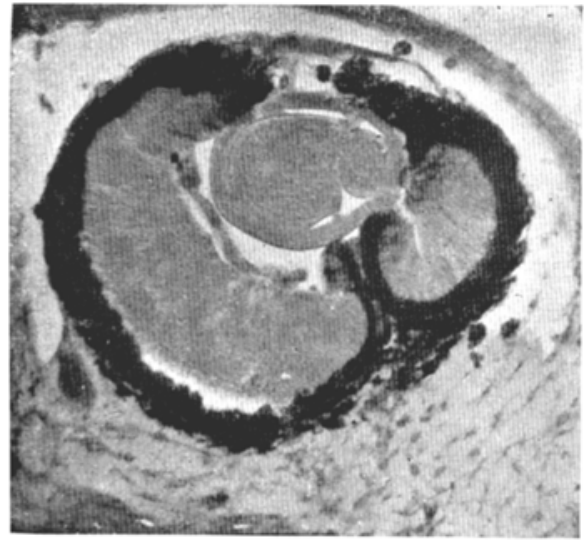

Fig. 14

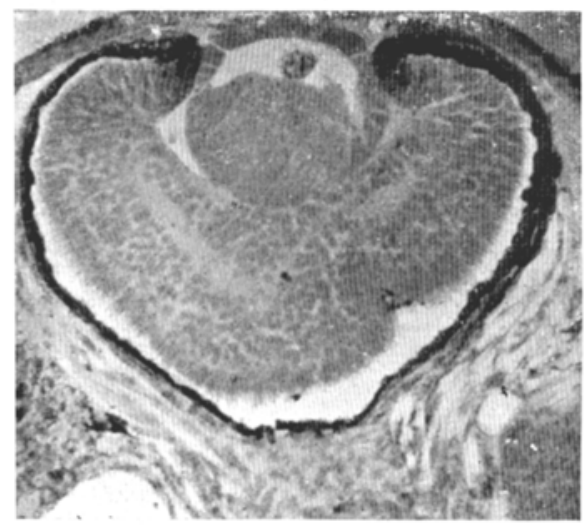

Fig. 15

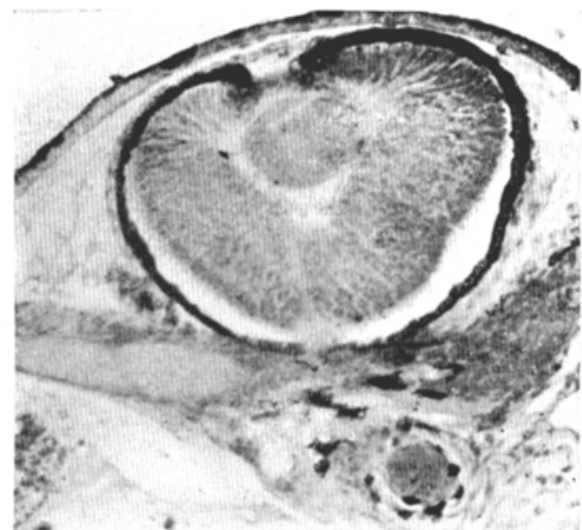

Fig. 16

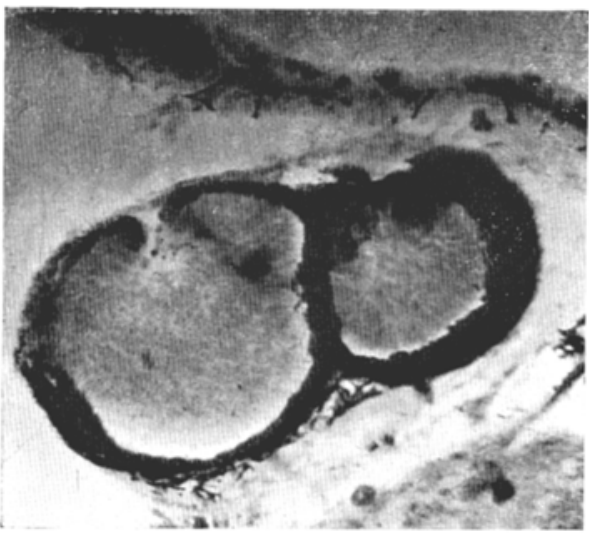

Fig. 17

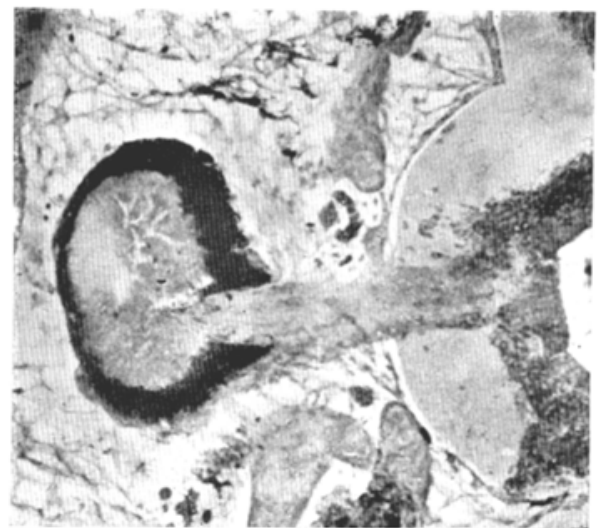

Fig. 18

nastast.

Verlag von Wilhelm Engelmann in Leipzig und Berlin. 